\title{
Super-Sample CMB Lensing
}

\author{
Alessandro Manzotti, ${ }^{1}$ Wayne $\mathrm{Hu},{ }^{1}$ and Aurélien Benoit-Lévy ${ }^{2}$ \\ ${ }^{1}$ Kavli Institute for Cosmological Physics, Department of Astronomy \& Astrophysics, \\ Enrico Fermi Institute, University of Chicago, Chicago, Illinois 60637, U.S.A. \\ ${ }^{2}$ Department of Physics and Astronomy, University College London, London WC1E 6BT, United Kingdom
}

\begin{abstract}
Lensing of the CMB by modes that are larger than the size of the survey dilates intrinsic scales in the temperature and polarization fields and coherently shifts their observed power spectra with respect to the ensemble or all-sky mean. The effect can be simply encapsulated as a contribution to the power spectrum covariance matrix in accordance with the lensing trispectrum or as an additional parameter, the mean convergence in the field, for parameter estimation. It should be included for upcoming surveys that precisely measure acoustic polarization features deep into the damping tail at multipoles of $\ell \gtrsim 1500$ with less than $10 \%$ of sky. Its omission may lead to seemingly conflicting values for the angular scale of the sound horizon which may then provide erroneous cosmological parameters when compared to baryon acoustic oscillation measurements.
\end{abstract}

\section{INTRODUCTION}

Gravitational lensing of the CMB is rapidly becoming both a useful tool for cosmology and a necessary component to model carefully in the statistical analysis of temperature and polarization anisotropy [1-7]. In particular, as first detections of CMB lensing in the $E$ and $B$ mode polarization power spectra become high significance measurements, it will become important to model non-Gaussian correlations induced by lensing when characterizing their statistical errors [8, 9].

For surveys that cover a large fraction of sky, the main contributions to non-Gaussian errors come from the fact that the sample variance of the lensing power and the unlensed CMB fields correlate power in the observed spectra across a wide range of multipoles 9]. However the next generation of surveys will focus on deep polarization sensitive measurements on small patches of sky. Here lensing by modes with wavelengths larger than the survey modify the observed power spectrum in the sub-survey modes.

Even a small amount of super-sample lensing can produce a significant effect, since all sub-survey band power measurements covary. In particular, lensing shifts the angular scale of the well-measured CMB acoustic peaks out as far in multipole moment that they can be measured. We call this effect super-sample covariance (SSC) following Ref. [10] for the same effect in large-scale structure [11] that similarly shifts the scale of baryon acoustic oscillations [12]. Indeed it is also this same modulation of small-scale anisotropy modes by long-wavelength lensing modes that enables lensing reconstruction techniques and squeezed bispectrum measurements of integrated-SachsWolfe effect correlation with lensing [13 16].

In this paper, we study the effect of lensing-induced SSC on CMB power spectrum measurements for small patches of sky. In $\sqrt{\Pi \text { II }}$ we derive the band power covariance matrix for temperature and polarization measurements and discuss the origin of SSC in the squeezed lensing trispectrum. We give a simple criteria for when SSC must be included in parameter estimation in III and discuss these results in $\$ \mathrm{IV}$.

\section{SUPER-SAMPLE COVARIANCE}

We introduce the SSC effect from lensing by supersample modes for temperature band power measurements in a finite sample in $\overline{\mathrm{IIA}}$. In $\$ \mathrm{IIB}$, we derive its form directly from the temperature trispectrum and in $\$ \mathrm{IIC}$ we give the complete expressions for temperature and polarization band power covariance.

\section{A. Temperature Field}

We begin by considering the covariance of temperature power spectra estimators due to lensing of the CMB in a finite survey of angular area $A$. A finite survey effectively measures the underlying temperature fluctuation field $T$ through a mask or window

$$
T_{W}(\boldsymbol{\theta})=T(\boldsymbol{\theta}) W(\boldsymbol{\theta})
$$

where $W(\boldsymbol{\theta})=1$ in the survey region and 0 otherwise. As we shall review in more detail in $8 \mathrm{IIB}$ in harmonic space the effect of the window is to convolve the fields or correlate band powers, destroying independent information for multipoles separated by $\Delta \ell \lesssim \ell_{W}=2 \pi / \theta_{W}$ where $\theta_{W} \sim \sqrt{A}$ is the angular dimension of the survey.

There are also physical effects that correlate band powers across $\Delta \ell \gg 2 \pi / \theta_{W}$ from gravitational lensing. For simplicity, in this paper we shall work exclusively in this wide bin limit rather than deconvolve the window which would obscure the underlying physical effects. These come about because two widely separated bands still jointly respond to the same modes in lensing potential. As such the covariance matrix for power estimates $\hat{C}_{\ell_{i}}$ in bands of width $\Delta \ell_{i} \gg \ell_{W}$ in the absence of instrument 
noise takes the form

$$
\begin{aligned}
\operatorname{Cov}_{\ell_{i} \ell_{j}} \equiv & \left\langle\hat{C}_{\ell_{i}} \hat{C}_{\ell_{j}}\right\rangle-\left\langle\hat{C}_{\ell_{i}}\right\rangle\left\langle\hat{C}_{\ell_{j}}\right\rangle \\
= & \frac{2 C_{\ell_{i}}^{2}}{\left(2 \ell_{i}+1\right) \Delta \ell_{i} f_{\mathrm{sky}}} \delta_{i j}+\frac{\partial \ell_{i}^{2} C_{\ell_{i}}}{\partial \ln \ell_{i}} \frac{\partial \ell_{j}^{2} C_{\ell_{j}}}{\partial \ln \ell_{j}} \frac{\sigma_{\kappa}^{2}}{\ell_{i}^{2} \ell_{j}^{2}} \\
& +\sum_{L}\left[\frac{\partial C_{\ell_{i}}}{\partial C_{L}^{\phi \phi}} \operatorname{Cov}_{L L}^{\phi \phi, \phi \phi} \frac{\partial C_{\ell_{j}}}{\partial C_{L}^{\phi \phi}}\right],
\end{aligned}
$$

where $C_{L}^{\phi \phi}$ is the power spectrum of the lensing potential $\phi$ and $\sigma_{\kappa}$ is the rms fluctuation in the associated convergence field $\kappa=-\nabla^{2} \phi / 2$ in the finite survey area

$$
\sigma_{\kappa}^{2}=\frac{1}{A^{2}} \sum_{L M}\left|W_{L M}\right|^{2} \frac{L^{2}(L+1)^{2}}{4} C_{L}^{\phi \phi} .
$$

Here $W_{L M}$ is the harmonic transform of the window. Finally the sample variance of $\hat{C}_{L}^{\phi \phi}$ in the survey area is given in the Gaussian approximation as

$$
\operatorname{Cov}_{L L}^{\phi \phi, \phi \phi}=\frac{2}{(2 L+1) f_{\text {sky }}}\left[C_{L}^{\phi \phi}\right]^{2} .
$$

The first term in Eq. (2) is the usual connected or Gaussian contribution. As we shall see explicitly in the trispectrum derivation of $\overline{I I B}$ the $f_{\text {sky }}$ or survey area factor $A=4 \pi f_{\text {sky }}$ represents the fact that within the band $\Delta \ell_{i}$ only modes separated by more than $\ell_{W}$ are independent due to convolution by the window.

The second term is linear in $C_{L}^{\phi \phi}$ and represents the effect of the mean convergence in the field. Since the mean convergence dilates the whole field, the power per logarithmic interval simply shifts in scale. In Eq. 2 we represent this power in the flat sky limit as $\ell^{2} C_{\ell} / 2 \pi$. While this approximation requires corrections for the curved sky if $\ell \lesssim 60$, the covariance induced by the shift is much smaller than the Gaussian variance term here and the error in the approximation has negligible impact. For $\sigma_{\kappa} \ll 1$, the covariance then takes the form of the second term. Following Ref. [10, we call this effect a supersample covariance (SSC) since the modes involved in producing the average convergence are on scales larger than the survey.

Since this rms convergence $\sigma_{\kappa}$ rapidly declines with survey area, the SSC effect was omitted in the all-sky analysis of Ref. [9]. Instead they introduced the third term in Eq. 2 which is higher order in $C_{L}^{\phi \phi}$ to describe the covariance produced by sampling fluctuations in the lensing potential power rather than individual modes. Since this response is very smooth in $L$, we have omitted binning in $L$ for notational clarity. This Gaussian sample variance of Eq. (4), like the first term, also scales with $f_{\text {sky. }}^{-1}$.

Thus the relative contribution of the SSC compared with other terms scales as $\sigma_{\kappa}^{2} f_{\text {sky }}$ and its dependence on $f_{\text {sky }}$ or $A$ is the relevant quantity to compute. For example if we consider a circular cap of polar angle $\theta_{W}$ then

$$
W_{L M}=\sqrt{\frac{\pi}{2 L+1}}\left[P_{L-1}(x)-P_{L+1}(x)\right] \delta_{M 0},
$$

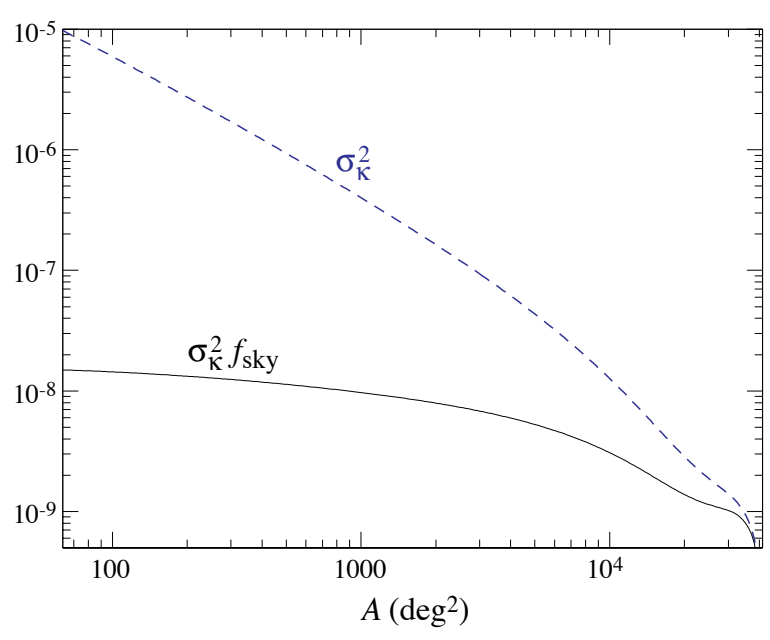

FIG. 1. Variance in the mean convergence $\sigma_{\kappa}^{2}$ in a circular patch of sky of area $A . \sigma_{\kappa}^{2}$ itself falls nearly as white noise (blue dashed line) leaving the quantity relevant for comparing terms in the power spectrum covariance $\sigma_{\kappa}^{2} f_{\text {sky }}$ (black, solid line) weakly dependent on survey area.

where $P_{L}$ is the Legendre polynomial with $x=\cos \theta_{W}$. Note that

$$
\frac{4 \pi}{A^{2}(2 L+1)} \sum_{M}\left|W_{L M}\right|^{2}=\left\{\begin{array}{ll}
1, & L \ll \ell_{W} \\
0, & L \gg \ell_{W}
\end{array},\right.
$$

where $A=2 \pi(1-x)$. As expected, the window function imposes a low pass filter on the total convergence power near the scale $\ell_{W}$. Since $C_{L}^{\phi \phi}$ is approximately proportional to $L^{-4}$, the convergence power is nearly white and $\sigma_{\kappa}^{2} \propto \ell_{W}^{2} \propto f_{\text {sky }}^{-1}$. Thus

$$
\sigma_{\kappa}^{2} f_{\text {sky }} \approx \text { const. }
$$

and the importance of the SSC term is very weakly dependent on the survey size. We quantify these considerations in Fig. 1 for a 6 parameter flat $\Lambda \mathrm{CDM}$ model that best fits the Planck $+\mathrm{WP}+$ lensing data combination [17]:

$$
\begin{aligned}
& \left\{\Omega_{c} h^{2}, \Omega_{b} h^{2}, 100 \theta_{*}, \tau, n_{s}, 10^{9} A_{s}\right\} \\
& \quad=\{0.118,0.0223,1.04167,0.0947,0.968,2.215\},
\end{aligned}
$$

where $\theta_{*}$ is the angular size of the sound horizon which implies $h=0.682$ and we also follow the Planck analysis in fixing $\Omega_{\nu} h^{2}=6.45 \times 10^{-4}$ for consistency with neutrino oscillation experiments. Note that we include here only contributions for $L \geq 2$, which causes the sharp decline in $\sigma_{\kappa}^{2} f_{\text {sky }}$ as $f_{\text {sky }} \rightarrow 1$.

\section{B. Trispectrum}

The SSC term in Eq. (2) can be more rigorously derived from the CMB trispectrum 18 following Ref. [10]. For simplicity, we employ the flat sky approximation in 
this section so that multipoles represent wavenumbers of a Fourier transform. As discussed in the previous section, this should be a good approximation for band powers where the covariance is important even if the survey itself is large enough to require a curved sky approach. In Fourier space, the window convolves modes in the spectrum

$$
T_{W}(\ell)=\int \frac{d^{2} \ell^{\prime}}{(2 \pi)^{2}} W\left(\ell-\ell^{\prime}\right) T\left(\ell^{\prime}\right) .
$$

We can define an estimator of the temperature fluctuation field as

$$
\hat{C}_{\ell_{i}}=\frac{1}{A} \int_{\ell \in \ell_{i}} \frac{d^{2} \ell}{A_{\ell}} T_{W}(\ell) T_{W}(-\ell),
$$

where the integral is over modes in some band around $\ell_{i}$ and $A_{\ell}=2 \pi \ell \Delta \ell$. Its expectation value

$$
\left\langle\hat{C}_{\ell_{i}}\right\rangle=\frac{1}{A} \int_{\ell \in \ell_{i}} \frac{d^{2} \ell}{A_{\ell}} \int \frac{d^{2} \ell^{\prime}}{(2 \pi)^{2}}\left|W\left(\ell^{\prime}\right)\right|^{2} C_{\left|\ell-\ell^{\prime}\right|}
$$

is unbiased so long as $C_{\ell}$ has no features on scales smaller than $\Delta \ell_{i}$ since

$$
\int \frac{d^{2} \ell^{\prime}}{(2 \pi)^{2}}\left|W\left(\ell^{\prime}\right)\right|^{2}=A .
$$

Using the fact that the power spectrum and trispectrum, or connected 4-pt function, are defined as

$$
\begin{aligned}
\left\langle T\left(\boldsymbol{\ell}_{1}\right) T\left(\boldsymbol{\ell}_{2}\right)\right\rangle & =(2 \pi)^{2} \delta\left(\boldsymbol{\ell}_{12}\right) C_{\ell_{1}}, \\
\left\langle T\left(\boldsymbol{\ell}_{1}\right) T\left(\boldsymbol{\ell}_{2}\right) T\left(\boldsymbol{\ell}_{3}\right) T\left(\boldsymbol{\ell}_{4}\right)\right\rangle_{c} & =(2 \pi)^{2} \delta\left(\boldsymbol{\ell}_{1234}\right) T\left(\boldsymbol{\ell}_{1}, \boldsymbol{\ell}_{2}, \boldsymbol{\ell}_{3}, \boldsymbol{\ell}_{4}\right) .
\end{aligned}
$$

where here and throughout $\ell_{1 \ldots n}=\ell_{1}+\ldots \ell_{n}$, we can divide the resulting covariance of the estimators into two pieces

$$
\operatorname{Cov}_{\ell_{i} \ell_{j}}=\mathrm{G}_{\ell_{i} \ell_{j}}+\mathrm{NG}_{\ell_{i} \ell_{j}} .
$$

The first term is the disconnected or Gaussian contribution of Eq. 2)

$$
\mathrm{G}_{\ell_{i} \ell_{j}}=\frac{(2 \pi)^{2}}{A A_{\ell}} 2 C_{\ell_{i}}^{2} \delta_{i j}
$$

as can be seen from taking $A=4 \pi f_{\text {sky }}$. This also defines the number of effectively independent $\ell$-modes

$$
N_{\text {modes }}=\frac{A_{\ell} A}{(2 \pi)^{2}}=\frac{A_{\ell}}{\ell_{W}^{2}}
$$

corresponding to the $\Delta \ell<\ell_{W}$ criteria of the previous section.

The second term comes from the trispectrum

$$
\begin{aligned}
\mathrm{NG}_{\ell_{i} \ell_{j}}= & \frac{1}{A^{2}} \int_{\ell \in \ell_{i}} \frac{d^{2} \ell}{A_{\ell}} \int_{\ell^{\prime} \in \ell_{j}} \frac{d^{2} \ell^{\prime}}{A_{\ell^{\prime}}} \\
& \int\left[\prod_{a=1}^{4} \frac{d^{2} L_{a}}{(2 \pi)^{2}} W\left(\mathbf{L}_{a}\right)\right](2 \pi)^{2} \delta\left(\mathbf{L}_{1234}\right) \\
& T\left(\boldsymbol{\ell}+\mathbf{L}_{1},-\boldsymbol{\ell}+\mathbf{L}_{2}, \ell^{\prime}+\mathbf{L}_{3},-\boldsymbol{\ell}+\mathbf{L}_{4}\right) .
\end{aligned}
$$

In the limit that $\ell_{i} \gg L_{i}$, we can relabel $\boldsymbol{\ell}+\mathbf{L}_{1} \rightarrow \boldsymbol{\ell}, \boldsymbol{\ell}^{\prime}+$ $\mathbf{L}_{3} \rightarrow \boldsymbol{\ell}^{\prime}$, and the delta function condition sets $\mathbf{L}=\boldsymbol{\ell}_{12}=$ $-\ell_{34}$. Since $W^{2}(\boldsymbol{\theta})=W(\boldsymbol{\theta})$, the convolution theorem

$$
\int \frac{d^{2} L_{1}}{(2 \pi)^{2}} W\left(\mathbf{L}_{1}\right) W\left(\mathbf{L}-\mathbf{L}_{1}\right)=W(\mathbf{L})
$$

can be used to express

$$
\begin{aligned}
\mathrm{NG}_{\ell_{i} \ell_{j}}= & \frac{1}{A^{2}} \int_{\ell \in \ell_{i}} \frac{d^{2} \ell}{A_{\ell}} \int_{\ell^{\prime} \in \ell_{j}} \frac{d^{2} \ell^{\prime}}{A_{\ell^{\prime}}} \int \frac{d^{2} L}{(2 \pi)^{2}}|W(\mathbf{L})|^{2} \\
& T\left(\ell,-\ell+\mathbf{L}, \ell^{\prime},-\ell^{\prime}-\mathbf{L}\right) .
\end{aligned}
$$

Note that the window connects multipoles that are separated by less than its fundamental mode $\ell_{W}$ and so the covariance no longer depends only on degenerate quadrilaterals through $T\left(\boldsymbol{\ell},-\boldsymbol{\ell}, \boldsymbol{\ell}^{\prime},-\boldsymbol{\ell}^{\prime}\right)$ but rather squeezed quadrilaterals of Eq. (19).

To linear order in $C_{\ell}^{\phi \phi}$, the lensing trispectrum is given by 18

$$
T\left(\ell_{1}, \ell_{2}, \ell_{3}, \ell_{4}\right)=C_{\ell_{1}} C_{\ell_{3}} C_{\ell_{12}}^{\phi \phi}\left(\ell_{12} \cdot \ell_{1}\right)\left(\ell_{34} \cdot \ell_{3}\right)+\text { perm. }
$$

where "perm." means all permutations of the $\boldsymbol{\ell}_{i}$. In the relevant squeezed quadrilateral limit $L \ll \ell_{1}$, we can expand

$$
\ell_{2} \approx \ell_{1}-\frac{\ell_{1} \cdot \ell_{12}}{\ell_{1}}
$$

so that

$$
C_{\ell_{2}} \approx C_{\ell_{1}}-\left.\frac{\partial C_{\ell}}{\partial \ln \ell}\right|_{\ell_{1}} \frac{\ell_{1} \cdot \ell_{12}}{\ell_{1}^{2}}
$$

and rewrite

$$
\ell_{12} \cdot \ell_{2}=-\ell_{12} \cdot \ell_{1}+\ell_{12}^{2}
$$

and similarly for $1 \rightarrow 3$ and $2 \rightarrow 4$. With these replacements in Eq. 19, we obtain

$$
\mathrm{NG}_{\ell_{i} \ell_{j}}=\frac{\partial \ell_{i}^{2} C_{\ell_{i}}}{\partial \ln \ell_{i}} \frac{\partial \ell_{j}^{2} C_{\ell_{j}}}{\partial \ln \ell_{j}} \frac{\sigma_{\kappa}^{2}}{\ell_{i}^{2} \ell_{j}^{2}},
$$

where

$$
\sigma_{\kappa}^{2}=\frac{1}{A^{2}} \int \frac{d^{2} L}{(2 \pi)^{2}} \frac{L^{4}}{4} C_{L}^{\phi \phi}|W(\mathbf{L})|^{2}
$$

which is the flat-sky limit of Eq. (3). Thus the trispectrum to linear order in $C_{L}^{\phi \phi}$ yields the SSC term of Eq. (2). Because this term vanishes for all-sky measurements where $L \rightarrow 0$, or more properly becomes indistinguishable from a change in the background cosmology, Ref. 9] found that the dominant term is higher order in $C_{L}^{\phi \phi}$. Here we consider the SSC effect for smaller survey fields. 


\section{Polarization}

The full covariance matrix of temperature and $E, B$ polarization power spectra takes a similar form

$$
\begin{aligned}
\operatorname{Cov}_{\ell_{i} \ell_{j}}^{W X, Y Z} & \equiv\left\langle\hat{C}_{\ell_{i}}^{W X} \hat{C}_{\ell_{j}}^{Y Z}\right\rangle-\left\langle\hat{C}_{\ell_{i}}^{W X}\right\rangle\left\langle\hat{C}_{\ell_{j}}^{Y Z}\right\rangle \\
& =\mathrm{G}_{\ell_{i} \ell_{j}}^{W X, Y Z}+\mathrm{NG}_{\ell_{i} \ell_{j}}^{W X, Y Z},
\end{aligned}
$$

where $\{W, X, Y, Z\} \in\{T, E, B\}$. The Gaussian contribution is

$$
\mathrm{G}_{\ell_{i} \ell_{j}}^{W X, Y Z}=\frac{C_{\ell_{i}}^{W Y} C_{\ell_{i}}^{X Z}+C_{\ell_{i}}^{W Z} C_{\ell_{i}}^{X Y}}{\left(2 \ell_{i}+1\right) \Delta \ell_{i} f_{\text {sky }}} \delta_{i j},
$$

where $C_{\ell}^{T T}=C_{\ell}$, and the SSC or linear in $C_{L}^{\phi \phi}$ term is

$$
\mathrm{NG}_{\ell_{i} \ell_{j}}^{W X, Y Z}=\frac{\partial \ell_{i}^{2} C_{\ell_{i}}^{W X}}{\partial \ln \ell_{i}} \frac{\partial \ell_{j}^{2} C_{\ell_{j}}^{Y Z}}{\partial \ln \ell_{j}} \frac{\sigma_{\kappa}^{2}}{\ell_{i}^{2} \ell_{j}^{2}}+\mathcal{O}\left(C_{L}^{\phi \phi}\right)^{2} .
$$

For the cases involving $T T, E E$, and $T E$, this expression can be directly derived from the leading order trispectrum [14, 19] in the same way as in the previous section. For $B B$ from lensing, the contribution is higher than leading order since $C_{\ell}^{B B}$ is itself $\mathcal{O}\left(C_{L}^{\phi \phi}\right)$. Its form can be inferred from the fact that the average convergence in the field lenses CMB polarization that is itself lensed into $B$ modes by small scale perturbations. This logic parallels that of the refinement [15, 16] of lensing reconstruction estimators [13, 20] where terms that are higher order in lensing are accounted for by replacing the unlensed CMB power spectra with the lensed CMB power spectra.

For completeness, we include the $\mathcal{O}\left(C_{L}^{\phi \phi}\right)^{2}$ terms introduced in Ref. 9. For the $B B$ power spectrum,

$$
\begin{aligned}
\mathrm{NG}_{\ell_{i} \ell_{j}}^{B B, B B}= & \ldots+\sum_{L} \frac{\partial C_{\ell_{i}}^{B B}}{\partial C_{L}^{\tilde{E} \tilde{E}}} \operatorname{Cov}_{L L}^{\tilde{E} \tilde{E}, \tilde{E} \tilde{E}} \frac{\partial C_{\ell_{j}}^{B B}}{\partial C_{L}^{\tilde{E} \tilde{E}}} \\
& +\sum_{L} \frac{\partial C_{\ell_{i}}^{B B}}{\partial C_{L}^{\phi \phi}} \operatorname{Cov}_{L L}^{\phi \phi, \phi \phi} \frac{\partial C_{\ell_{j}}^{B B}}{\partial C_{L}^{\phi \phi}},
\end{aligned}
$$

where tildes denote the unlensed power spectrum and its covariance follows the Gaussian prescription of Eq. 27. Here and below "..." denotes the linear SSC term of Eq. 28. For $W X=B B$ and $\{Y, Z\} \in\{T, E\}$,

$$
\begin{aligned}
\mathrm{NG}_{\ell_{i} \ell_{j}}^{B B, Y Z}= & \ldots+\sum_{L} \frac{\partial C_{\ell_{i}}^{B B}}{\partial C_{L}^{\tilde{E} \tilde{E}}} \operatorname{Cov}_{L L}^{\tilde{E} \tilde{E}, \tilde{Y} \tilde{Z}} \frac{\partial C_{\ell_{j}}^{Y Z}}{\partial C_{L}^{\tilde{Y} \tilde{Z}}} \\
& +\sum_{L} \frac{\partial C_{\ell_{i}}^{B B}}{\partial C_{L}^{\phi \phi}} \operatorname{Cov}_{L L}^{\phi \phi, \phi \phi} \frac{\partial C_{\ell_{j}}^{Y Z}}{\partial C_{L}^{\phi \phi}},
\end{aligned}
$$

and for $\{W, X\} \in\{T, E\}$ as well

$$
\mathrm{NG}_{\ell_{i} \ell_{j}}^{W X, Y Z}=\ldots+\sum_{L} \frac{\partial C_{\ell_{i}}^{W X}}{\partial C_{L}^{\phi \phi}} \operatorname{Cov}_{L L}^{\phi \phi, \phi \phi} \frac{\partial C_{\ell_{j}}^{Y Z}}{\partial C_{L}^{\phi \phi}} .
$$

The impact of these other terms on parameter estimation was addressed in Ref. 9 and so we focus on that of the SSC term next.

\section{SIGNAL VS. NOISE}

A simple way to assess the impact of the SSC effect is to consider it as part of the signal rather than the noise. As noise, SSC introduces a covariance because the whole power spectrum dilates with the average convergence in the field $\bar{\kappa}$ whose variance $\sigma_{\kappa}^{2}$ makes band powers covary field-to-field. As signal, $\bar{\kappa}$ is simply added to the cosmological parameters $\mathbf{p}$ in the likelihood analysis of an individual field

$$
\hat{C}_{\ell}^{X Y}(\mathbf{p} ; \kappa)=C_{\ell}^{X Y}(\mathbf{p})-\frac{\partial \ell^{2} C_{l}^{X Y}(\mathbf{p})}{\partial \ln \ell} \frac{\bar{\kappa}}{\ell^{2}},
$$

which is subject to a Gaussian prior given $\sigma_{\kappa}^{2}$.

To determine whether the SSC effect is important to include for any cosmological parameter set, it is sufficient to test its impact on an artificial fully-degenerate parameter $\mathbf{p}=s$ that also dilates scales in the power spectra

$$
C_{\ell}^{X Y}(s)=\bar{C}_{\ell}^{X Y}+\frac{\partial \ell^{2} \bar{C}_{l}^{X Y}}{\partial \ln \ell} \frac{s}{\ell^{2}} .
$$

If for a given experiment $\sigma_{s}<\sigma_{\kappa}$ then $\bar{\kappa}$ should in principle be marginalized in the analysis or SSC included in the covariance. Here $\bar{C}_{\ell}^{\mathrm{XY}}$ represents some fixed fiducial power spectrum which we take to be the Planck best fit model described in \$II A. It is interesting to note that if the inflationary power spectrum contains features of width $\Delta \ell$ which are finer than the acoustic spacing $\ell_{W}<\Delta \ell \lesssim 300$, then the impact of SSC can be greatly enhanced 21.

We can estimate $\sigma_{s}$ with the Fisher technique. For an arbitrary set of parameters $\mathbf{p}, \sigma_{p_{\mu}}^{2}=\left(\mathbf{F}^{-1}\right)_{\mu \mu}$ where the Fisher matrix

$$
F_{\mu \nu}=\sum_{i j} \sum_{W X, Y Z} \frac{\partial C_{\ell_{i}}^{W X}}{\partial p_{\mu}}\left(\mathrm{G}_{\ell_{i} \ell_{j}}^{W X}\right)^{-1} \frac{\partial C_{\ell_{j}}^{Y Z}}{\partial p_{\nu}}
$$

Here we specialize to a single parameter $s$ and the Gaussian covariance of Eq. 27). This is the Gaussian sample variance limit.

In Fig. 2, we show the quantity $\sigma_{s}^{2} f_{\text {sky }}$ in the limit of bins that are much finer than the acoustic scale $\Delta \ell \ll 300$ summed out to $\ell_{i} \leq \ell_{\max }$. This should be compared with $\sigma_{\kappa}^{2} f_{\text {sky }}$ which we also show for comparison for the range of $400 \leq A\left(\mathrm{deg}^{2}\right) \leq 4000$ and a circular patch from Fig. 1. Note that for sample variance limited measurements of all power spectra, the two cross between $1200 \lesssim \ell_{\max } \lesssim 1600$. Thus the SSC effect should be modeled for surveys which are sample variance limited to at least these multipoles.

We also show in Fig. 2 the individual contributions for each type of spectrum. The measurement of $s$ mainly reflects the $E E$ and $T E$ power spectra given the more prominent acoustic features in these spectra. For the $T T$ spectrum alone, this crossing point is pushed to $\ell_{\max } \gtrsim$ 2800 and a real survey is likely to be foreground limited before this point. For the $B B$ spectrum there is negligible 
impact even for sample variance limited measurements given its smooth form.

To further make these considerations concrete we can include instrument noise $N_{\ell}^{X Y}$ by replacing in the Gaussian covariance of Eq. 27

$$
C_{\ell}^{X Y} \rightarrow C_{\ell}^{X Y}+N_{\ell}^{X Y},
$$

where

$$
N_{\ell}^{T T}=\frac{1}{2} N_{\ell}^{E E}=\frac{1}{2} N_{\ell}^{B B}=\Delta_{T}^{2} e^{\ell(\ell+1) \theta_{\mathrm{FWHM}}^{2} / 8 \ln 2},
$$

with all other $N_{\ell}^{X Y}=0$. Here $\Delta_{T}$ is the noise level in $\mu \mathrm{K}$-radians and $\theta_{\mathrm{FWHM}}$ is the full-width half-max of the beam in radians. We also restrict the bands to $\ell_{\max } \leq$ 2000, 3000 beyond which the primary anisotropy may be too difficult to extract from foregrounds and secondaries. In Tab. I. we show that for a typical second generation $(2 \mathrm{G})$ and third generation $(3 \mathrm{G})$ survey, the SSC variance $\sigma_{\kappa}^{2}$ exceeds $\sigma_{s}^{2}$ by a factor of $2-3$ for $\ell_{\max }=2000-3000$. This indicates that the SSC effect should be included in the analysis of these surveys.

While $\sigma_{\kappa}^{2} / \sigma_{s}^{2}$ determines whether SSC is important at all, its impact on physical cosmological parameters depends on whether or not the given parameter is degenerate with a pure shift $s$. We can use the Fisher technique to study the SSC impact on parameter estimation by appending $\bar{\kappa}$ to these parameters and adding a prior

$$
F_{\mu \nu}^{\text {prior }}=\sigma_{\kappa}^{-2} \delta_{\bar{\kappa}, \mu} \delta_{\bar{\kappa}, \nu}
$$

The impact of SSC can be determined by comparing the change in parameter errors upon marginalizing $\bar{\kappa}$ vs fixing $\bar{\kappa}=0$.

In the $\Lambda$ CDM parameter space defined by the parameters in Eq. 8), only the angular size of the sound horizon $\theta_{*}$ is nearly degenerate with $s$ and hence only its variance degrades significantly. In Tab. [1] we quantify this degradation as

$$
R_{\theta_{*}} \equiv \frac{\sigma_{\ln \theta_{*}}^{2}}{\left.\sigma_{\ln \theta_{*}}^{2}\right|_{\bar{\kappa}=0}} \leq 1+\frac{\sigma_{\kappa}^{2}}{\sigma_{s}^{2}} .
$$

The inequality follows from the fact that at low $\ell$ the power spectrum is not dominated by acoustic features and more importantly, at high $\ell$ lensing effects enter so that $\sigma_{\ln \theta_{*}}^{2} \geq \sigma_{s}^{2}$. Even so, the degradation in the variance of $\theta_{*}$ is $R_{\theta_{*}} \sim 2-3$ for the $2 \mathrm{G}$ and $3 \mathrm{G}$ experiments. We have also conducted this test using the covariance approach by adding only the SSC term of Eq. (28) to the Gaussian error and omitting $\bar{\kappa}$ as a parameter. This method obtains nearly identical results for the degradation in $\sigma_{\ln \theta_{*}}^{2}$.

For estimation purposes, it is useful to note that given the degeneracy between $\ln \theta_{*}$ and $\kappa$ this degradation factor is to good approximation

$$
R_{\theta_{*}} \approx 1+\frac{\sigma_{\kappa}^{2}}{\left.\sigma_{\ln \theta_{*}}^{2}\right|_{\bar{\kappa}=0}} .
$$

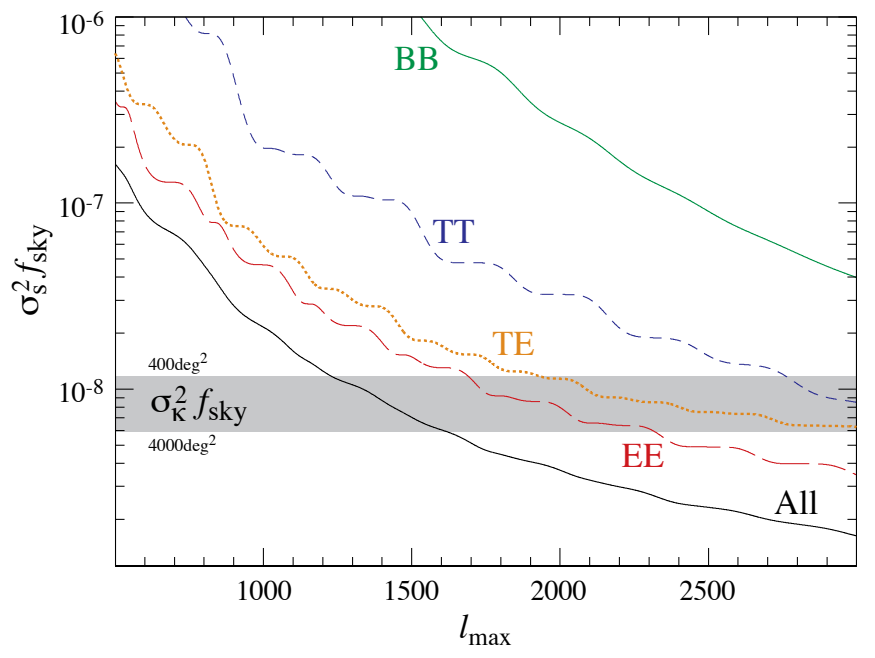

FIG. 2. Gaussian sample variance limited constraints on a parameter $s$ that dilates the CMB power spectra similar to $\ln \theta_{*}$ in the acoustic regime. When the expected variance $\sigma_{s}^{2}$ is comparable to or smaller that of the mean convergence in the field $\sigma_{\kappa}^{2}$, the SSC effect should be included in the analysis. For a wide range of sky coverage $400 \leq A\left(\operatorname{deg}^{2}\right) \leq 4000, \sigma_{\kappa}^{2} f_{\text {sky }}$ is within the shaded band indicating that SSC is important for such measurements if they are sample variance limited to at least $\ell_{\max } \sim 1200-1600$.

For example, this expression approximates values in Tab. I at the $1 \%$ level.

We can use this approximation to estimate the SSC effect for the Planck data. Taking the smallest patch of sky $f_{\text {sky }}=0.37$ from which the power spectrum is measured to maximize the SSC effect and crudely assuming a circular patch, we obtain $\sigma_{\kappa} \sim 7 \times 10^{-5}$. This should be compared with the quoted precision on the acoustic scale $\sigma_{\ln \theta_{*}}=5.8 \times 10^{-4}$. We thus conclude that the SSC effect is unimportant for the analysis of the Planck data.

On the other hand, its omission in the $2 \mathrm{G}$ and $3 \mathrm{G}$ cases may lead to seemingly discrepant results for $\theta_{*}$ when compared with either Planck results or with each other. Discrepant results on $\theta_{*}$ have little impact on CMB measurements of fundamental parameters through the distance to recombination due to uncertainties in the physical scale of the sound horizon. For example, the errors on the Hubble constant in a flat $\Lambda$ CDM model are dominated by errors on $\Omega_{c} h^{2}$ not $\theta_{*}$. However they may lead to erroneous results when compared to measurements of the same scale through baryon acoustic oscillations.

\section{DISCUSSION}

We have explored the effect of CMB lensing by super sample modes on power spectra measurements within a finite sky sample. The resulting field-to-field fluctuations in the mean convergence dilates the intrinsic scales in the survey and coherently shifts the measured power spectra with respect to the ensemble or all-sky mean. The effect 


\begin{tabular}{|c|c|c|c|c|c|c|c|}
\hline & $\Delta_{T}$ & $\theta_{\mathrm{FWHM}}$ & $A$ & \multicolumn{2}{|c|}{$1+\sigma_{\kappa}^{2} / \sigma_{s}^{2}\left(\ell_{\max }\right)$} & \multicolumn{2}{|c|}{$R_{\theta_{*}}\left(\ell_{\max }\right)$} \\
& $\left(\mu \mathrm{K}^{\prime}\right)$ & $(\operatorname{arcmin})$ & $\left(\mathrm{deg}^{2}\right)$ & 2000 & 3000 & 2000 & 3000 \\
\hline $2 \mathrm{G}$ & 12 & $1^{\prime}$ & 500 & 2.7 & 3.5 & 2.4 & 2.6 \\
\hline $3 \mathrm{G}$ & 3.5 & $1^{\prime}$ & 2500 & 2.8 & 4.1 & 2.5 & 3.0 \\
\hline
\end{tabular}

TABLE I. Relative importance of SSC for example experimental specifications. $1+\sigma_{\kappa}^{2} / \sigma_{s}^{2}$ gives the maximal degradation in variance for a parameter that is degenerate with the shift due to lensing whereas $R_{\theta_{*}}$ gives the same for the angular size of the sound horizon.

can be simply encapsulated as an extra fully covarying SSC contribution to the covariance matrix, as described by the lensing trispectrum, or as an additional parameter, the mean convergence, that must be included in parameter estimation.

By comparing the ability of a given experiment to measure an overall change in angular scale to the expected rms mean convergence, we provide a simple test for when the SSC effect should be included in data analysis. This criterion is met for surveys that precisely measure acoustic polarization features deep into the damping tail at multipoles of $\ell \gtrsim 1500$ with less than $\sim 10 \%$ of sky. If this effect is omitted in the analysis then different surveys may measure seemingly conflicting values for the angular scale of the sound horizon $\theta_{*}$ any one of which may then provide erroneous fundamental cosmological parameters when compared to external measurements of the same standard ruler from baryon acoustic oscillations.

\section{ACKNOWLEDGMENTS}

We thank Clarence Chang for providing the SPTPol and SPT-3G target specifications for our $2 \mathrm{G}$ and $3 \mathrm{G}$ test cases and Kendrick Smith for useful conversations. WH was supported by by U.S. Dept. of Energy contract DEFG02-13ER41958, the Kavli Institute for Cosmological Physics at the University of Chicago through grants NSF PHY-0114422 and NSF PHY-0551142 and the David and Lucile Packard Foundation. ABL Acknowledges support from the Leverhulme Trust and the Science and Technology Facilities Council.
[1] S. Das, B. D. Sherwin, P. Aguirre, J. W. Appel, J. R. Bond, et al., Phys.Rev.Lett. 107, 021301 (2011), arXiv:1103.2124 [astro-ph.CO],

[2] S. Das, T. Louis, M. R. Nolta, G. E. Addison, E. S. Battistelli, et al., (2013), arXiv:1301.1037 [astro-ph.CO],

[3] R. Keisler, C. Reichardt, K. Aird, B. Benson, L. Bleem, et al., Astrophys.J. 743, 28 (2011), arXiv:1105.3182 [astro-ph.CO].

[4] A. van Engelen, R. Keisler, O. Zahn, K. Aird, B. Benson, et al., Astrophys.J. 756, 142 (2012), arXiv:1202.0546 [astro-ph.CO].

[5] D. Hanson et al. (SPTpol Collaboration), Phys.Rev.Lett. 111, 141301 (2013), arXiv:1307.5830 [astro-ph.CO].

[6] P. Ade et al. (Polarbear Collaboration), (2013), arXiv:1312.6646 [astro-ph.CO].

[7] P. Ade et al. (Planck Collaboration), (2013), arXiv:1303.5077 [astro-ph.CO].

[8] K. M. Smith, W. Hu, and M. Kaplinghat, Phys. Rev. D 74, 123002 (2006), arXiv:astro-ph/0607315.

[9] A. Benoit-Levy, K. M. Smith, and W. Hu, Phys.Rev. D86, 123008 (2012), arXiv:1205.0474 [astro-ph.CO].

[10] M. Takada and W. Hu, Phys.Rev. D87, 123504 (2013), arXiv:1302.6994 [astro-ph.CO],

[11] A. J. Hamilton, C. D. Rimes, and R. Scoccimarro, Mon.Not.Roy.Astron.Soc. 371, 1188 (2006), arXiv:astro- ph/0511416 [astro-ph]

[12] Y. Li, W. Hu, and M. Takada, (2014), arXiv:1401.0385 [astro-ph.CO]

[13] W. Hu, Astrophys.J. 557, L79 (2001), arXiv:astro$\mathrm{ph} / 0105424$ [astro-ph].

[14] T. Okamoto and W. Hu, Phys.Rev. D66, 063008 (2002) arXiv:astro-ph/0206155 [astro-ph]

[15] D. Hanson, A. Challinor, G. Efstathiou, and P. Bielewicz, Phys.Rev. D83, 043005 (2011), arXiv:1008.4403 [astro-ph.CO].

[16] A. Lewis, A. Challinor, and D. Hanson, JCAP 1103, 018 (2011), arXiv:1101.2234 [astro-ph.CO]

[17] Planck Explanatory Supplement

[18] W. Hu, Phys.Rev. D64, 083005 (2001), arXiv:astro$\mathrm{ph} / 0105117$ [astro-ph].

[19] W. Hu, Phys.Rev. D62, 043007 (2000), arXiv:astroph/0001303 [astro-ph],

[20] T. Okamoto and W. Hu, Phys. Rev. D 67, 083002 (2003), arXiv:astro-ph/0301031

[21] V. Miranda and W. Hu, (2013), arXiv:1312.0946 [astroph.CO] 